\title{
New Tribonacci recurrence relations and addition formulas
}

\section{Kunle Adegoke $^{1}$, Adenike Olatinwo ${ }^{2}$ and Winning Oyekanmi ${ }^{3}$}

${ }^{1}$ Department of Physics and Engineering Physics, Obafemi Awolowo University 220005 Ile-Ife, Nigeria

e-mail: adegoke00@gmail.com

${ }^{2}$ Department of Physics and Engineering Physics, Obafemi Awolowo University 220005 Ile-Ife, Nigeria e-mail: solakunle711@yahoo.com

${ }^{3}$ Department of Physics and Engineering Physics, Obafemi Awolowo University 220005 Ile-Ife, Nigeria e-mail: winningteam90@yahoo.com

Received: 13 March 2020

Abstract: Only one three-term recurrence relation, namely, $W_{r}=2 W_{r-1}-W_{r-4}$, is known for the generalized Tribonacci numbers, $W_{r}, r \in \mathbb{Z}$, defined by $W_{r}=W_{r-1}+W_{r-2}+W_{r-3}$ and $W_{-r}=W_{-r+3}-W_{-r+2}-W_{-r+1}$, where $W_{0}, W_{1}$ and $W_{2}$ are given, arbitrary integers, not all zero. Also, only one four-term addition formula is known for these numbers, which is $W_{r+s}=T_{s-1} W_{r-1}+\left(T_{s-1}+T_{s-2}\right) W_{r}+T_{s} W_{r+1}$, where $\left(T_{r}\right)_{r \in \mathbb{Z}}$ is the Tribonacci sequence, a special case of the generalized Tribonacci sequence, with $W_{0}=T_{0}=0$ and $W_{1}=W_{2}=T_{1}=T_{2}=1$. In this paper we discover three new three-term recurrence relations and two identities from which a plethora of new addition formulas for the generalized Tribonacci numbers may be discovered. We obtain a simple relation connecting the Tribonacci numbers and the Tribonacci-Lucas numbers. Finally, we derivequadratic and cubic recurrence relations for the generalized Tribonacci numbers. Keywords: Tribonacci number, Tribonacci-Lucas number, Recurrence relation.

2010 Mathematics Subject Classification: 11B39, 11 B37.

\section{Introduction}

For $r \geq 3$, we define the generalized Tribonacci numbers $W_{r}$ by the third order recurrence relation: 


$$
W_{r}=W_{r-1}+W_{r-2}+W_{r-3},
$$

where $W_{0}, W_{1}$ and $W_{2}$ are arbitrary integers. By writing $W_{r-1}=W_{r-2}+W_{r-3}+W_{r-4}$ and subtracting this from relation (1), we see that $W_{r}$ also obeys the useful three-term recurrence

$$
W_{r}=2 W_{r-1}-W_{r-4} .
$$

Extension of the definition of the generalized Tribonacci numbers to negative subscripts is provided by writing identity (2) as $W_{r+4}=2 W_{r+3}-W_{r}$; so that

$$
W_{-r}=2 W_{-r+3}-W_{-r+4} .
$$

Well known examples of $W_{r}$ are the Tribonacci sequence, $\left(T_{r}\right), r \in \mathbb{Z}$, for which $W=T$, $W_{0}=T_{0}=0, W_{1}=W_{2}=T_{1}=T_{2}=1$ and the Tribonacci-Lucas sequence, $\left(K_{r}\right), r \in \mathbb{Z}$, for which $W=K, W_{0}=K_{0}=3, W_{1}=K_{1}=1, W_{2}=K_{2}=3$. Table 1 shows the first few Tribonacci and Tribonacci-Lucas numbers for $-20 \leq r \leq 24$.

\begin{tabular}{cccccccccc}
$r$ & -20 & -19 & -18 & -17 & -16 & -15 & -14 & -13 & -12 \\
\hline$T_{r}$ & -56 & 159 & -103 & 0 & 56 & -47 & 9 & 18 & -20 \\
$K_{r}$ & 795 & -571 & 47 & 271 & -253 & 65 & 83 & -105 & 43
\end{tabular}

\begin{tabular}{cccccccccc}
$r$ & -11 & -10 & -9 & -8 & -7 & -6 & -5 & -4 & -3 \\
\hline$T_{r}$ & 7 & 5 & -8 & 4 & 1 & -3 & 2 & 0 & -1 \\
$K_{r}$ & 21 & -41 & 23 & 3 & -15 & 11 & -1 & -5 & 5
\end{tabular}

\begin{tabular}{cccccccccc}
$r$ & -2 & -1 & 0 & 1 & 2 & 3 & 4 & 5 & 6 \\
\hline$T_{r}$ & 1 & 0 & 0 & 1 & 1 & 2 & 4 & 7 & 13 \\
$K_{r}$ & -1 & -1 & 3 & 1 & 3 & 7 & 11 & 21 & 39
\end{tabular}

\begin{tabular}{cccccccccc}
$r$ & 7 & 8 & 9 & 10 & 11 & 12 & 13 & 14 & 15 \\
\hline$T_{r}$ & 24 & 44 & 81 & 149 & 274 & 504 & 927 & 1705 & 3136 \\
$K_{r}$ & 71 & 131 & 241 & 443 & 815 & 1499 & 2757 & 5071 & 9327
\end{tabular}

\begin{tabular}{cccccccccc}
$r$ & 16 & 17 & 18 & 19 & 20 & 21 & 22 & 23 & 24 \\
\hline$T_{r}$ & 5768 & 10609 & 19513 & 35890 & 66012 & 121415 & 223317 & 410744 & 755476 \\
$K_{r}$ & 17155 & 31553 & 58035 & 106743 & 196331 & 361109 & 664183 & 1221623 & 2246915
\end{tabular}

Table 1. The first few Tribonacci and Tribonacci-Lucas numbers.

The following references contain useful information on the properties of the Tribonacci numbers and related: $[1,3,5,6,8,9]$. Various congruence properties of Tribonacci sequences are discussed in the recent paper by Atanassova [2].

Among other interesting results, we found the following three-term recurrence relations which are presumably new: $W_{r-16}=-103 W_{r}+56 W_{r+1}, 2 W_{r-17}=9 W_{r}-103 W_{r-4}$ and $W_{r-14}=9 W_{r+2}-56 W_{r-1}$. 
We also found a simple relation linking the Tribonacci numbers and the Tribonacci-Lucas numbers:

$$
K_{r-2}=5 T_{r-1}-T_{r+1}
$$

\section{Linear recurrence relations and addition formulas}

Theorem 2.1. The following identity holds for integers $a, b, c, d$ and $e$ :

$$
\begin{aligned}
& \left(T_{a-c} T_{c-b} T_{b-a}+T_{b-c} T_{c-a} T_{a-b}\right) W_{d+e} \\
& =\left(T_{b-c} T_{c-a} T_{e-b}-T_{b-c} T_{c-b} T_{e-a}+T_{c-b} T_{b-a} T_{e-c}\right) W_{d+a} \\
& \quad+\left(T_{a-c} T_{c-b} T_{e-a}-T_{a-c} T_{c-a} T_{e-b}+T_{c-a} T_{a-b} T_{e-c}\right) W_{d+b} \\
& \quad+\left(T_{b-c} T_{a-b} T_{e-a}-T_{a-b} T_{b-a} T_{e-c}+T_{a-c} T_{b-a} T_{e-b}\right) W_{d+c}
\end{aligned}
$$

Proof. We seek to express a generalized Tribonacci number as a linear combination of three Tribonacci numbers. Let

$$
W_{d+e}=f_{1} T_{e-a}+f_{2} T_{e-b}+f_{3} T_{e-c},
$$

where $a, b, c, d$ and $e$ are arbitrary integers and the coefficients $f_{1}, f_{2}$ and $f_{3}$ are to be determined. Setting $e=a, e=b$ and $e=c$, in turn, we obtain three simultaneous equations:

$$
W_{d+a}=f_{2} T_{a-b}+f_{3} T_{a-c}, \quad W_{d+b}=f_{1} T_{b-a}+f_{3} T_{b-c}, \quad W_{d+c}=f_{1} T_{c-a}+f_{2} T_{c-b} .
$$

The identity of Theorem 2.1 is established by solving these equations for $f_{1}, f_{2}$ and $f_{3}$ and substituting the solutions into identity (5).

Corollary 2.1.1. The following identities hold for integers $r$ and $s$ :

$$
\begin{gathered}
4 W_{r+s}=2 T_{s-1} W_{r-4}+\left(T_{s+4}-7 T_{s}\right) W_{r}+4 T_{s} W_{r+1}, \\
4 W_{r+s}=2 T_{s-4} W_{r-1}+\left(4 T_{s+1}-7 T_{s}\right) W_{r}+T_{s} W_{r+4}, \\
W_{r+s}=T_{s-1} W_{r-1}+\left(T_{s+1}-T_{s}\right) W_{r}+T_{s} W_{r+1}, \\
4 W_{r+s}=T_{s-4} W_{r-4}+\left(T_{s+4}-11 T_{s}\right) W_{r}+T_{s} W_{r+4}, \\
W_{r+s}=\left(T_{s+1}-2 T_{s}-T_{s-2}\right) W_{r-1}+\left(T_{s+1}-2 T_{s}\right) W_{r}+T_{s} W_{r+2} .
\end{gathered}
$$

Proof. To derive identity (6), set $a=r-4, b=r, c=r+1, d=0$ and $e=r+s$ in the identity of Theorem 2.1. The proof of (7)—(10) is similar, (see Table 2).

\begin{tabular}{cccccc} 
Identity & $a$ & $b$ & $c$ & $d$ & $e$ \\
\hline (6) & $r-4$ & $r$ & $r+1$ & 0 & $r+s$ \\
\hline (7) & $r+4$ & $r$ & $r-1$ & 0 & $r+s$ \\
\hline$(8)$ & $r-1$ & $r$ & $r+1$ & 0 & $r+s$ \\
\hline
\end{tabular}

\begin{tabular}{cccccc} 
Identity & $a$ & $b$ & $c$ & $d$ & $e$ \\
\hline (9) & $r+4$ & $r$ & $r-4$ & 0 & $r+s$ \\
\hline (10) & $r+2$ & $r-1$ & $r$ & 0 & $r+s$ \\
\hline
\end{tabular}

Table 2. Appropriate substitutions in the identity of Theorem 2.1 to obtain identities (6) - (10) of Corollary 2.1.1. 
Note that identity (8) can be written in the familiar form

$$
W_{r+s}=T_{s-1} W_{r-1}+\left(T_{s-1}+T_{s-2}\right) W_{r}+T_{s} W_{r+1} .
$$

Evaluating identity (6) at $s=-3, s=-16$ and at $s=-17$, in turn, we find the following three term recurrence relations for the generalized Tribonacci numbers:

$$
\begin{gathered}
W_{r-3}=2 W_{r}-W_{r+1}, \\
W_{r-16}=-103 W_{r}+56 W_{r+1}
\end{gathered}
$$

and

$$
2 W_{r-17}=9 W_{r}-103 W_{r-4} .
$$

Evaluating identity (10) at $s=-14$ produces yet another three-term recurrence relation for the generalized Tribonacci numbers:

$$
W_{r-14}=9 W_{r+2}-56 W_{r-1} .
$$

Note that by interchanging $r$ and $s$ in each case and making use of the defining recurrence relation for $W$ and $T$, the identities (6) - (10) can also be written

$$
\begin{gathered}
4 W_{r+s}=2 W_{s-1} T_{r-4}+\left(W_{s+4}-7 W_{s}\right) T_{r}+4 W_{s} T_{r+1}, \\
4 W_{r+s}=2 W_{s-4} T_{r-1}+\left(4 W_{s+1}-7 W_{s}\right) T_{r}+W_{s} T_{r+4}, \\
W_{r+s}=W_{s-1} T_{r-1}+\left(W_{s+1}-W_{s}\right) T_{r}+W_{s} T_{r+1}, \\
4 W_{r+s}=W_{s-4} T_{r-4}+\left(W_{s+4}-11 W_{s}\right) T_{r}+W_{s} T_{r+4}, \\
W_{r+s}=\left(W_{s+1}-2 W_{s}-W_{s-2}\right) T_{r-1}+\left(W_{s+1}-2 W_{s}\right) T_{r}+W_{s} T_{r+2} .
\end{gathered}
$$

Evaluating identity (18) at $s=-2$ with $W=K$ gives

$$
K_{r-2}=5 T_{r-1}-T_{r+1}
$$

a three-term relation connecting the Tribonacci numbers and the Tribonacci-Lucas numbers.

Relations similar to (4) and (21) are also derived by Frontczak [4] and Komatsu [7].

Theorem 2.2. The following identity holds for integers $a, b, c, d$ and $e$ :

$$
\begin{aligned}
& \left\{K_{b-a-1} K_{a-b-1}+K_{b-a-1} K_{c-b-1} K_{a-c-1}+K_{c-a-1} K_{b-c-1} K_{a-b-1}\right. \\
& \left.+K_{c-a-1} K_{a-c-1}+K_{c-b-1} K_{b-c-1}-1\right\} W_{d+e} \\
& =\left\{\left(1-K_{c-b-1} K_{b-c-1}\right) K_{e-a}+\left(K_{b-a-1}+K_{c-a-1} K_{b-c-1}\right) K_{e-b}\right. \\
& \left.+\left(K_{c-a-1}+K_{b-a-1} K_{c-b-1}\right) K_{e-c}\right\} W_{d+a-1} \\
& +\left\{\left(1-K_{c-a-1} K_{a-c-1}\right) K_{e-b}+\left(K_{a-b-1}+K_{c-b-1} K_{a-c-1}\right) K_{e-a}\right. \\
& \left.+\left(K_{c-b-1}+K_{c-a-1} K_{a-b-1}\right) K_{e-c}\right\} W_{d+b-1} \\
& +\left\{\left(1-K_{b-a-1} K_{a-b-1}\right) K_{e-c}+\left(K_{b-c-1}+K_{b-a-1} K_{a-c-1}\right) K_{e-b}\right. \\
& \left.+\left(K_{a-c-1}+K_{b-c-1} K_{a-b-1}\right) K_{e-a}\right\} W_{d+c-1} .
\end{aligned}
$$


Proof. We wish to express a generalized Tribonacci number as a linear combination of three Tribonacci-Lucas numbers. Let

$$
W_{d+e}=f_{1} K_{e-a}+f_{2} K_{e-b}+f_{3} K_{e-c},
$$

where $a, b, c, d$ and $e$ are arbitrary integers and the coefficients $f_{1}, f_{2}$ and $f_{3}$ are to be determined. Setting $e=a-1, e=b-1$ and $e=c-1$, in turn, we obtain three simultaneous equations:

$$
\begin{gathered}
W_{d+a-1}=-f_{1}+f_{2} K_{a-b-1}+f_{3} K_{a-c-1}, \quad W_{d+b-1}=f_{1} K_{b-a-1}-f_{2}+f_{3} K_{b-c-1}, \\
W_{d+c-1}=f_{1} K_{c-a-1}+f_{2} K_{c-b-1}-f_{3} .
\end{gathered}
$$

The identity of Theorem 2.2 is obtained by solving these equations for $f_{1}, f_{2}$ and $f_{3}$ and substituting the solutions into identity (22).

Corollary 2.2.1. The following identities hold for integers $r$ and $s$ :

$$
\begin{aligned}
44 W_{r+s}= & \left(9 K_{s+3}-K_{s+5}+2 K_{s+1}\right) W_{r-6} \\
& +\left(9 K_{s+1}+K_{s+5}+2 K_{s+3}\right) W_{r-4}+\left(K_{s+3}+6 K_{s+5}-K_{s+1}\right) W_{r-2} \\
88 W_{r+s}=(8 & \left.K_{s+3}-30 K_{s-1}-2 K_{s-2}\right) W_{r} \\
+ & \left(K_{s+3}-K_{s-1}+8 K_{s-2}\right) W_{r-4}+\left(3 K_{s+3}+19 K_{s-1}+2 K_{s-2}\right) W_{r+1} \\
22 W_{r+s}=\left(5 K_{s-2}+\right. & \left.K_{s-1}+2 K_{s}\right) W_{r-1} \\
+\left(K_{s-2}-\right. & \left.2 K_{s-1}+7 K_{s}\right) W_{r}+\left(2 K_{s-2}+7 K_{s-1}+3 K_{s}\right) W_{r+1} \\
5060 W_{r+s}= & \left(-264 K_{s-3}+K_{s+9}-1079 K_{s-1}\right) W_{r-10} \\
& +\left(-4279 K_{s-3}+21 K_{s+9}-21394 K_{s-1}\right) W_{r} \\
& +\left(6325 K_{s-1}+1265 K_{s-3}\right) W_{r+2}
\end{aligned}
$$

and

$$
\begin{aligned}
5060 W_{r+s}= & \left(-264 K_{s-11}+1265 K_{s+1}-4279 K_{s-1}\right) W_{r-2} \\
& +\left(-1079 K_{s-11}+6325 K_{s+1}-21394 K_{s-1}\right) W_{r} \\
& +\left(21 K_{s-1}+K_{s-11}\right) W_{r+10} .
\end{aligned}
$$

Identities (23) - (27) can also be written as follows.

$$
\begin{aligned}
& 44 W_{r+s}=\left(9 W_{s+3}-W_{s+5}+2 W_{s+1}\right) K_{r-6} \\
&+\left(9 W_{s+1}+W_{s+5}+2 W_{s+3}\right) K_{r-4}+\left(W_{s+3}+6 W_{s+5}-W_{s+1}\right) K_{r-2} \\
& 88 W_{r+s}=\left(8 W_{s+3}-30 W_{s-1}-2 W_{s-2}\right) K_{r} \\
&+\left(W_{s+3}-W_{s-1}+8 W_{s-2}\right) K_{r-4}+\left(3 W_{s+3}+19 W_{s-1}+2 W_{s-2}\right) K_{r+1} \\
& 22 W_{r+s}=\left(5 W_{s-2}+W_{s-1}+2 W_{s}\right) K_{r-1} \\
&+\left(W_{s-2}-2 W_{s-1}+7 W_{s}\right) K_{r}+\left(2 W_{s-2}+7 W_{s-1}+3 W_{s}\right) K_{r+1} \\
& 5060 W_{r+s}=\left(-264 W_{s-3}+W_{s+9}-1079 W_{s-1}\right) K_{r-10} \\
&+\left(-4279 W_{s-3}+21 W_{s+9}-21394 W_{s-1}\right) K_{r} \\
&+\left(6325 W_{s-1}+1265 W_{s-3}\right) K_{r+2}
\end{aligned}
$$


and

$$
\begin{aligned}
5060 W_{r+s}= & \left(-264 W_{s-11}+1265 W_{s+1}-4279 W_{s-1}\right) K_{r-2} \\
& +\left(-1079 W_{s-11}+6325 W_{s+1}-21394 W_{s-1}\right) K_{r} \\
& +\left(21 W_{s-1}+W_{s-11}\right) K_{r+10} .
\end{aligned}
$$

\section{Quadratic relations}

Our goal in this section is to derive expressions involving only pure squares of generalized Tribonacci numbers. To achieve this we must be able to express the anticipated cross-terms such as $W_{r-1} W_{r}$ and $W_{r-1} W_{r-4}$ as squares of generalized Tribonacci numbers.

Rearranging identity (2) and squaring, we have

$$
\begin{gathered}
4 W_{r-1} W_{r}=4 W_{r-1}^{2}-W_{r-4}^{2}+W_{r}^{2}, \\
4 W_{r-1} W_{r-4}=4 W_{r-1}^{2}+W_{r-4}^{2}-W_{r}^{2}
\end{gathered}
$$

and

$$
2 W_{r} W_{r-4}=4 W_{r-1}^{2}-W_{r-4}^{2}-W_{r}^{2} .
$$

Rearranging identity (2) and multiplying through by $4 W_{r-3}$ to obtain

$$
8 W_{r-1} W_{r-3}=4 W_{r} W_{r-3}+4 W_{r-4} W_{r-3},
$$

and using identities (33) and (34) to resolve the right hand side gives

$$
8 W_{r-1} W_{r-3}=4 W_{r}^{2}+2 W_{r-3}^{2}-W_{r+1}^{2}+4 W_{r-4}^{2}-W_{r-7}^{2} .
$$

Multiplying through identity (2) by $4 W_{r-5}$ to obtain

$$
4 W_{r} W_{r-5}=8 W_{r-1} W_{r-5}-4 W_{r-4} W_{r-5},
$$

which, with the use of (33) and (35), translates to

$$
4 W_{r} W_{r-5}=16 W_{r-2}^{2}-8 W_{r-5}^{2}-4 W_{r-1}^{2}+W_{r-8}^{2}-W_{r-4}^{2} .
$$

Rearranging identity (2), shifting index $r$ and multiplying through by $2 W_{r}$ gives

$$
2 W_{r} W_{r-8}=4 W_{r} W_{r-5}-2 W_{r} W_{r-4},
$$

from which, using (35) and (39), we get

$$
2 W_{r} W_{r-8}=16 W_{r-2}^{2}-8 W_{r-5}^{2}-8 W_{r-1}^{2}+W_{r-8}^{2}+W_{r}^{2} .
$$

Rearranging identity (13) and squaring, we have

$$
\begin{gathered}
112 W_{r-17} W_{r}=W_{r-17}^{2}+3136 W_{r}^{2}-10609 W_{r-1}^{2} \\
11536 W_{r-1} W_{r}=-W_{r-17}^{2}+3136 W_{r}^{2}+10609 W_{r-1}^{2},
\end{gathered}
$$


and

$$
206 W_{r-17} W_{r-1}=-W_{r-17}^{2}-10609 W_{r-1}^{2}+3136 W_{r}^{2} .
$$

Rearranging and squaring identity (14), we have

$$
\begin{aligned}
& 36 W_{r-17} W_{r}=4 W_{r-17}^{2}+81 W_{r}^{2}-10609 W_{r-4}^{2}, \\
& 1854 W_{r} W_{r-4}=81 W_{r}^{2}+10609 W_{r-4}^{2}-4 W_{r-17}^{2}
\end{aligned}
$$

and

$$
412 W_{r-17} W_{r-4}=-4 W_{r-17}^{2}-10609 W_{r-4}^{2}+81 W_{r}^{2} .
$$

Finally, squaring and rearranging identity (15) produces

$$
\begin{aligned}
& 18 W_{r-17} W_{r-1}=W_{r-17}^{2}+81 W_{r-1}^{2}-3136 W_{r-4}^{2}, \\
& 1008 W_{r-1} W_{r-4}=81 W_{r-1}^{2}+3136 W_{r-4}^{2}-W_{r-17}^{2}
\end{aligned}
$$

and

$$
112 W_{r-17} W_{r-4}=-W_{r-17}^{2}-3136 W_{r-4}^{2}+81 W_{r-1}^{2} .
$$

Theorem 3.1. The following identities hold for any integer $r$ :

$$
\begin{gathered}
252 W_{r}^{2}-927 W_{r-1}^{2}+2884 W_{r-4}^{2}-W_{r-17}^{2}=0, \\
W_{r}^{2}-2 W_{r-1}^{2}-3 W_{r-2}^{2}-6 W_{r-3}^{2}+W_{r-4}^{2}+W_{r-6}^{2}=0 .
\end{gathered}
$$

Proof. Eliminating $W_{r-1} W_{r}$ between identities (33) and (43) proves identity (51). To prove identity (52), write $W_{r}-W_{r-1}=W_{r-2}+W_{r-3}$, square both sides and use the identity (33) to resolve the cross-products $W_{r} W_{r-1}$ and $W_{r-2} W_{r-3}$.

Substituting for $W_{r-17}^{2}$ from the identity of Theorem 3.1 into identities (42), (44) and (50), we have the following simpler versions of these identities:

$$
\begin{gathered}
4 W_{r-17} W_{r}=-412 W_{r-1}^{2}+103 W_{r-4}^{2}+121 W_{r}^{2}, \\
W_{r-17} W_{r-1}=-47 W_{r-1}^{2}-14 W_{r-4}^{2}+14 W_{r}^{2}
\end{gathered}
$$

and

$$
4 W_{r-17} W_{r-4}=36 W_{r-1}^{2}-215 W_{r-4}^{2}-9 W_{r}^{2} .
$$

Next we show how to express the square of a Tribonacci-Lucas number in terms of squares of Tribonacci numbers.

Theorem 3.2. The following identity holds for any integer $r$ :

$$
4 K_{r}^{2}=5 T_{r+5}^{2}-20 T_{r+4}^{2}+4 T_{r+3}^{2}+90 T_{r+1}^{2}-20 T_{r}^{2}+5 T_{r-3}^{2} .
$$

Proof. Square identity (21) and use identity (37) to eliminate the cross-term. 
Theorem 3.3. The following identities hold for integers $r$ and $s$ :

$$
\begin{aligned}
16 W_{r+s}^{2}=- & \left(T_{s}+T_{s+4}\right)\left(-T_{s+4}+2 T_{s-1}+7 T_{s}\right) W_{r}^{2}+4 T_{s-1} T_{s} W_{r-7}^{2} \\
+ & 2 T_{s-1}\left(-T_{s+4}-9 T_{s}+2 T_{s-1}\right) W_{r-4}^{2}-2 T_{s}\left(T_{s+4}-7 T_{s}+2 T_{s-1}\right) W_{r-3}^{2} \\
+ & 8 T_{s-1}\left(T_{s}+T_{s+4}\right) W_{r-1}^{2}+2 T_{s}\left(T_{s}+T_{s+4}\right) W_{r+1}^{2} \\
16 W_{r+s}^{2}=4 & \left.42 T_{s}-T_{s+1}\right)\left(7 T_{s}-T_{s-4}-4 T_{s+1}\right) W_{r}^{2}+4 T_{s-4}\left(2 T_{s}-T_{s+1}\right) W_{r-4}^{2} \\
& -4 T_{s-4}\left(-T_{s-4}-4 T_{s+1}+9 T_{s}\right) W_{r-1}^{2}+16 T_{s-4} T_{s} W_{r+2}^{2} \\
- & 4 T_{s}\left(7 T_{s}+T_{s-4}-4 T_{s+1}\right) W_{r+3}^{2}+4 T_{s}\left(2 T_{s}-T_{s+1}\right) W_{r+4}^{2} \\
4 W_{r+s}^{2}= & -2\left(-T_{s+1}+T_{s}\right)\left(2 T_{s+2}-T_{s-1}\right) W_{r}^{2}-T_{s-1} T_{s} W_{r-5}^{2} \\
& +2 T_{s-1}\left(-T_{s+1}+T_{s}\right) W_{r-4}^{2}+2 T_{s}\left(-T_{s+1}+T_{s}\right) W_{r-3}^{2} \\
& +4 T_{s-1} T_{s} W_{r-2}^{2}+2 T_{s-1}\left(2 T_{s-1}+4 T_{s+1}-3 T_{s}\right) W_{r-1}^{2} \\
& +2 T_{s}\left(T_{s}+T_{s+1}\right) W_{r+1}^{2}-T_{s-1} T_{s} W_{r+3}^{2}+4 T_{s-1} T_{s} W_{r+2}^{2} .
\end{aligned}
$$

\section{Cubic recurrence relations}

Theorem 4.1. The following identity holds for integer $r$ :

$$
\begin{aligned}
& W_{r}^{3}-4 W_{r-1}^{3}-9 W_{r-2}^{3}-34 W_{r-3}^{3}+24 W_{r-4}^{3}-2 W_{r-5}^{3} \\
& \quad+40 W_{r-6}^{3}-14 W_{r-7}^{3}-W_{r-8}^{3}-2 W_{r-9}^{3}+W_{r-10}^{3}=0 .
\end{aligned}
$$

Proof. Setting $a=r-8, b=r, c=r-10, d=0$ and $e=r-s$ with $s \in\{1,2,3,4,5,6,7,9\}$ in the identity of Theorem 2.1, the following linear combinations are formed:

$$
\begin{aligned}
W_{r-1} & =\frac{37}{68} W_{r}-\frac{5}{68} W_{r-8}+\frac{1}{17} W_{r-10}, & W_{r-2} & =\frac{5}{17} W_{r}+\frac{3}{17} W_{r-8}+\frac{1}{17} W_{r-10}, \\
W_{r-3} & =\frac{11}{68} W_{r}-\frac{7}{68} W_{r-8}-\frac{2}{17} W_{r-10}, & W_{r-4} & =\frac{3}{34} W_{r}-\frac{5}{34} W_{r-8}+\frac{2}{17} W_{r-10}, \\
W_{r-5} & =\frac{3}{68} W_{r}+\frac{29}{68} W_{r-8}+\frac{1}{17} W_{r-10}, & W_{r-6} & =\frac{1}{34} W_{r}-\frac{13}{34} W_{r-8}-\frac{5}{17} W_{r-10}, \\
W_{r-7} & =\frac{1}{68} W_{r}-\frac{13}{68} W_{r-8}+\frac{6}{17} W_{r-10}, & W_{r-9} & =\frac{1}{68} W_{r}-\frac{81}{68} W_{r-8}-\frac{11}{17} W_{r-10} .
\end{aligned}
$$

The above $W_{r-i}, i \in\{1,2,3,4,5,6,7,9\}$ verify the identity of Theorem 4.1 .

Taking the cube in identities (12) and (13) and solving two simultaneous equations, we find

$$
155736 W_{r} W_{r-1}^{2}=-W_{r-17}^{3}+199305 W_{r-1}^{3}-161504 W_{r-4}^{3}+14112 W_{r}^{3}
$$

and

$$
77868 W_{r}^{2} W_{r-1}=-148526 W_{r-4}^{3}+27090 W_{r}^{3}-W_{r-17}^{3}+95481 W_{r-1}^{3} .
$$

Theorem 4.2. The following identity holds for integer $r$ :

$$
\begin{aligned}
& 11844 W_{r}^{3}+3 W_{r-19}^{3}+W_{r-17}^{3}+458556 W_{r-6}^{3} \\
& \quad+135548 W_{r-4}^{3}-442179 W_{r-3}^{3}-120204 W_{r-2}^{3}-43569 W_{r-1}^{3}=0 .
\end{aligned}
$$

Proof. Write the defining recurrence relation of the generalized Tribonacci numbers as $W_{r}-W_{r-1}=W_{r-2}+W_{r-3}$, take the cube of both sides and use identities (60) and (61) to remove cross-terms. 


\section{Conclusion}

We have derived many new recurrence relations for the generalized Tribonacci sequence. We discovered three new three-term recurrence relations and two identities from which numerous new addition formulas for generalized Tribonacci numbers may be discovered. We obtained a simple relation connecting the Tribonacci numbers and the Tribonacci-Lucas numbers. Finally, we derived quadratic and cubic recurrence relations for the generalized Tribonacci sequence.

\section{Acknowledgements}

Thanks are due to the two anonymous referees whose comments helped to improve the presentation.

\section{References}

[1] Adegoke, K. (2018). Weighted Tribonacci sums, arXiv:1804.06449[math.CA].

[2] Atanassova, L. (2019). A remark on the Tribonacci sequences, Notes on Number Theory and Discrete Mathematics, 25(3), 138-141.

[3] Feng, J. (2011). More identities on the Tribonacci numbers, Ars Combinatoria, 100, 73-78.

[4] Frontczak, R. (2018). Convolutions for generalized Tribonacci numbers and related results, International Journal of Mathematical Analysis, 12 (7), 307-324.

[5] Frontczak, R. (2018). Sums of Tribonacci and Tribonacci-Lucas numbers, International Journal of Mathematical Analysis, 12(1), 19-24.

[6] Gabai, H. (1970). Generalized Fibonacci $k$-sequences, The Fibonacci Quarterly, 8(1), $31-38$.

[7] Komatsu, T. (2011). On the sum of reciprocal Tribonacci numbers, Ars Combinatoria, 98, $447-459$.

[8] Shah, D. V. (2011). Some Tribonacci identities, Mathematics Today, 27, 1-9.

[9] Waddill, M. E., \& Sacks, L. (1967). Another generalized Fibonacci sequence, The Fibonacci Quarterly, 5(3), 209-222. 